Document downloaded from:

http://hdl.handle.net/10251/125102

This paper must be cited as:

Xu, S.; Chen, C.; Benítez López, J. (2018). Projections for generalized inverses. Linear and Multilinear Algebra. 66(8):1593-1605. https://doi.org/10.1080/03081087.2017.1364339

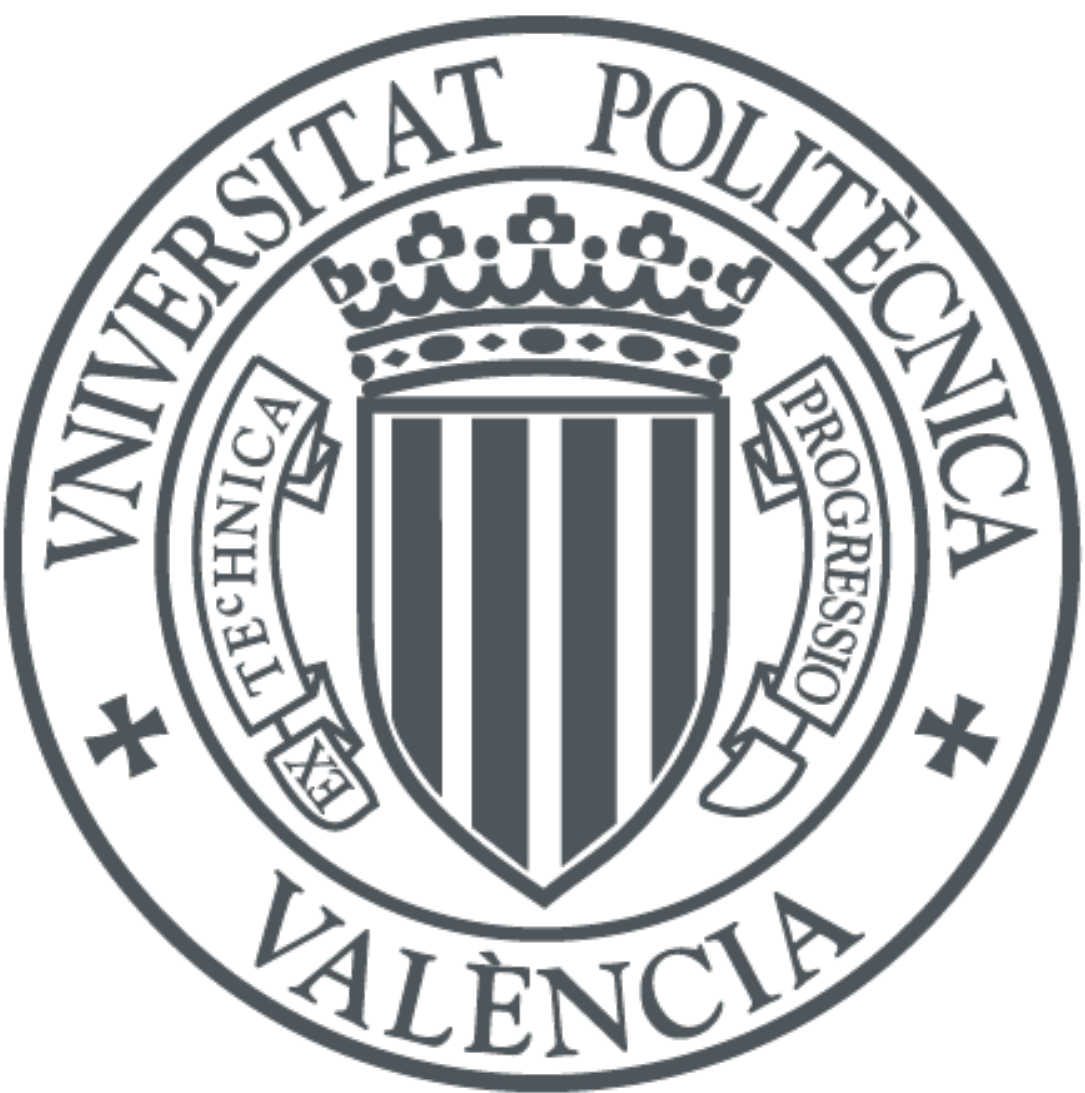

The final publication is available at

http://doi.org/10.1080/03081087.2017.1364339

Copyright Taylor \& Francis

Additional Information 


\title{
Projections for generalized inverses
}

\author{
Sanzhang Xu and Jianlong Chen*
}

School of Mathematics, Southeast University, Nanjing 210096, China

\section{Julio Benítez ${ }^{\dagger}$}

Universitat Politècnica de València, Instituto de Matemática Multidisciplinar, Valencia, 46022, Spain

\begin{abstract}
Let $R$ be a unital ring with involution. In Section 2, for given two core invertible elements $a, b \in R$, we investigate mainly the absorption law for the core inverse in virtue of the equality of the projections $a a^{\boxplus}$ and $b b^{\oplus}$. In Section 3, we study several relations concerning the projections $a^{\prime} a$ and $b b^{\prime}$, where $a^{\prime} \in a\{1,2,4\}$ and $b^{\prime} \in b\{1,2,3\}$. Some well-known results are extended to the $*$-reducing ring case. As an application, EP elements in a $*$-reducing ring are considered.
\end{abstract}

Key words: Projection, absorption law, core inverse, Moore-Penrose inverse.

AMS subject classifications: 15A09, 16W10, $16 \mathrm{U} 60$.

\section{Introduction}

Throughout this paper, $R$ will denote a unital ring with involution, i.e., a ring with unity 1 , and a mapping $a \mapsto a^{*}$ satisfying $\left(a^{*}\right)^{*}=a,(a b)^{*}=b^{*} a^{*}$ and $(a+b)^{*}=a^{*}+b^{*}$, for all $a, b \in R$. Let $a, x \in R$. If
(1) $a x a=a$,
(2) $x a x=x$,
(3) $(a x)^{*}=a x$,
(4) $(x a)^{*}=x a$,

then $x$ is called a Moore-Penrose inverse of $a$. If such an element $x$ exists, then it is unique and denoted by $a^{\dagger}$. The set of all Moore-Penrose invertible elements will be denoted by $R^{\dagger}$. Let $I \subset\{1,2,3,4\}$. An element $b \in R$ is called an $I$ inverse of $a \in R$ if equalities $i \in I$ of (1.1) hold. The set of all $I$ inverses of $a$ will be denoted by $a^{I}$, the element $a$ is $I$ invertible when $a^{I} \neq \varnothing$ and the set of all $I$ invertible elements will be denoted by $R^{I}$. Let $a \in R$. It can be easily proved that the set of elements $x \in R$ such that

$$
a x a=a, \quad x a x=x \quad \text { and } \quad a x=x a
$$

is empty or a singleton. If this set is a singleton, its unique element is called the group inverse of $a$ and denoted by $a^{\#}$. The set of all group invertible elements will be denoted by $R^{\#}$. The subset of $R$ of all invertible elements will be denoted by $R^{-1}$. We will also use the following notations: $a R=\{a x: x \in R\}, R a=\{x a: x \in R\},{ }^{\circ} a=\{x \in R: x a=0\}$ and $a^{\circ}=\{x \in R: a x=0\}$.

\footnotetext{
*E-mail: xusanzhang5222@126.com. Corresponding author, E-mail: jlchen@seu.edu.cn

${ }^{\dagger}$ E-mail: jbenitez@mat.upv.es
} 
The notion of the core inverse for a complex matrix was introduced by Baksalary and Trenkler [1]. In [12], Rakić et al. generalized the core inverse of a complex matrix to the case of an element in $R$. More precisely, let $a, x \in R$, if

$$
a x a=a, \quad x R=a R \quad \text { and } \quad R x=R a^{*},
$$

then $x$ is called a core inverse of $a$. If such an element $x$ exists, then it is unique and denoted by $a^{\boxplus}$. The set of all core invertible elements in $R$ will be denoted by $R^{\boxplus}$. Also, in [12] the authors defined a related inner inverse in a ring with an involution. If $a \in R$, then $x \in R$ is called a dual core inverse of $a$ if

$$
a x a=a, \quad x R=a^{*} R \text { and } R x=R a .
$$

If such an element $x$ exists, then it is unique and denoted by $a_{\circledast}$. The set of all dual core invertible elements in $R$ will be denoted by $R_{\circledast}$. It is evident that $a \in R^{\oplus}$ if and only if $a^{*} \in R_{\circledast}$, and in this case, one has $\left(a^{\oplus}\right)^{*}=\left(a^{*}\right)_{\circledast}$. More characterizations of elements to be core invertible by equations can be found in $[12,15]$.

An element $a \in R$ is said to be an EP element if $a \in R^{\dagger} \cap R^{\#}$ and $a^{\dagger}=a^{\#}[2,5]$. The set of all EP elements will be denoted by $R^{\mathrm{EP}}$. An element $p \in R$ is said to be a projection if $p^{2}=p=p^{*}$. We will use the notation $[a, b]=a b-b a$.

In [7, Proposition 6], for two complex matrices $A$ and $B$, Hartwig and Spindelböck explored equivalent conditions such that $A^{\dagger} A=B B^{\dagger}$. In [11, Theorem 2.3], for $a, b \in R^{\dagger}$, Patrício and Mendes investigated necessary and sufficient conditions such that $a a^{\dagger}=b b^{\dagger}$. If we take $c=b^{\dagger}$ in $a a^{\dagger}=b b^{\dagger}$, then by $\left(b^{\dagger}\right)^{\dagger}=b$, we have $a a^{\dagger}=c^{\dagger} c$, and therefore, this form is the same as $A^{\dagger} A=B B^{\dagger}$. In [7, 11], authors investigated the equality $a a^{\dagger}=b b^{\dagger}$ under the hypothesis $a, b \in R^{\dagger}$. Motivated by [7, 11], in Section 2, we discuss when the projections $a a^{\boxplus}$ and $b b^{\oplus}$ are equal, which is under the hypothesis $a, b \in R^{\boxplus}$. We will extend [7, Proposition 6] of complex matrices to the case of $*$-reducing rings in Section 3. In Theorem 3.6, when $a^{(1,2,4)} \in a^{\{1,2,4\}}$ and $b^{(1,2,3)} \in b^{\{1,2,3\}}$, we give some relationships between the projections $a^{(1,2,4)} a$ and $b b^{(1,2,3)}$. Theorem 3.6 will be useful in the sequel. In Theorem 3.9, we can see that the proof becomes simple with the aid of Theorem 3.6. Note that Theorem 3.9 is a generalization of known equivalent conditions for an EP element in a $*$-reducing ring.

\section{$2 \quad$ Core invertibility: the case $a a^{\boxplus}=b b^{\boxplus}$}

Before we investigate necessary and sufficient conditions such that two core invertible elements satisfy $a a^{\boxplus}=b b^{\boxplus}$, some auxiliary work should be done.

Proposition 2.1. Let $a, b \in R$. We have:

(1) If $a, b \in R^{\{1,3\}}$, then the following statements are equivalent:

(i) $a R=b R$;

(ii) $a a^{(1,3)}=b b^{(1,3)}$ for all $a^{(1,3)} \in a\{1,3\}$ and $b^{(1,3)} \in b\{1,3\}$;

(iii) $a a^{(1,3)}=b b^{(1,3)}$ for some $a^{(1,3)} \in a\{1,3\}$ and $b^{(1,3)} \in b\{1,3\}$. 
(2) If $a, b \in R^{\{1,4\}}$, then the following statements are equivalent:

(i) $R a=R b$;

(ii) $a^{(1,4)} a=b^{(1,4)} b$ for all $a^{(1,4)} \in a\{1,4\}$ and $b^{(1,4)} \in b\{1,4\}$;

(iii) $a^{(1,4)} a=b^{(1,4)} b$ for some $a^{(1,4)} \in a\{1,4\}$ and $b^{(1,4)} \in b\{1,4\}$.

Proof. Let us prove (1). (i) $\Rightarrow$ (ii). Suppose $a R=b R$, then $a=b x$ and $b=a y$ for some $x, y \in R$. Thus

$$
\begin{aligned}
& a=b x=b b^{(1,3)} b x=b b^{(1,3)} a \\
& b=a y=a a^{(1,3)} a y=a a^{(1,3)} b .
\end{aligned}
$$

Then we have

$$
\begin{aligned}
& b b^{(1,3)} \stackrel{\stackrel{(2.2)}{=}}{=} a a^{(1,3)} b b^{(1,3)}=\left(a a^{(1,3)} b b^{(1,3)}\right)^{*}=b b^{(1,3)} a a^{(1,3)} ; \\
& a a^{(1,3)} \stackrel{(2.1)}{=} b b^{(1,3)} a a^{(1,3)} .
\end{aligned}
$$

A combination of (2.3) and (2.4) implies $a a^{(1,3)}=b b^{(1,3)}$.

(ii) $\Rightarrow$ (iii) is obvious.

(iii) $\Rightarrow$ (i). Suppose exists $a^{(1,3)} \in a\{1,3\}$ and $b^{(1,3)} \in b\{1,3\}$ such that $a a^{(1,3)}=b b^{(1,3)}$. Then we have $a=a a^{(1,3)} a=b b^{(1,3)} a$ and $b=b b^{(1,3)} b=a a^{(1,3)} b$, thus $a R=b R$.

The proof of (2) is similar to the proof of (1).

In [12, Theorem 2.14], Rakić et al. proved that an element $a \in R^{\oplus}$ satisfies

$$
a a^{\oplus} a=a, \quad a^{\boxplus} a a^{\boxplus}=a^{\boxplus}, \quad\left(a a^{\boxplus}\right)^{*}=a a^{\oplus}, \quad a\left(a^{\oplus}\right)^{2}=a^{\oplus}, \quad a^{\oplus} a^{2}=a .
$$

Also, in [12, Theorem 2.15], they proved that an element $a \in R_{\circledast}$ satisfies

$$
a a_{\circledast} a=a, \quad a_{\oplus} a a_{\circledast}=a_{\oplus}, \quad\left(a_{\circledast} a\right)^{*}=a^{\oplus} a, \quad\left(a^{\oplus}\right)^{2} a=a_{\oplus}, \quad a^{2} a_{\circledast}=a .
$$

Thus a core invertible element is $\{1,2,3\}$-invertible and a dual core invertible element is $\{1,2,4\}$-invertible.

Two proofs of the next corollary can be found in [3, Proposition 1, Chapter 1] and in [12, Lemma 2.10]. We present another proof based on Proposition 2.1.

Corollary 2.2. Let $p, q \in R$ be two projections. Then $p R=q R$ if and only if $p=q$.

Proof. Since $p, q \in R$ are projections, then $p^{2}=p=p^{\dagger}$ and $q^{2}=q=q^{\dagger}$. By Proposition 2.1, $p R=q R$ implies $p p^{\dagger}=q q^{\dagger}$, that is $p=q$. The converse is clear.

In [11, Theorem 2.3], for $a, b \in R^{\dagger}$, the authors investigated equivalent conditions such that $a a^{\dagger}=b b^{\dagger}$. It is evident that the equality $x x^{\dagger} R=x R$ holds when $x \in R^{\dagger}$, thus the first item of next Corollary 2.3 follows from this observation and previous Corollary 2.2. The second item of next Corollary 2.3 follows from $x^{*} R=x^{\dagger} x R,(R x)^{*}=x^{*} R$, and previous Corollary 2.2. If $x \in R^{\#} \cap R^{\dagger}=R^{\boxplus} \cap R_{\circledast}$, by [12, Theorems 2.11 and 2.12] it follows that $x x^{\dagger}=x x^{\oplus}$ and $x^{\dagger} x=x_{\oplus} x$. The proof of next Corollary 2.3 only uses Proposition 2.1. Also, it is noteworthy that the characterization of $a a^{\boxplus}=b b^{\oplus}\left(a_{\circledast} a=b_{\oplus} b\right)$ is generalized because only $a, b \in R^{\oplus}\left(a, b \in R_{\circledast}\right.$, resp.) is used. 
Corollary 2.3. Let $a, b \in R$. Then we have:

(1) If $a, b \in R^{\dagger}$, then $a R=b R$ is equivalent to $a a^{\dagger}=b b^{\dagger}$;

(2) If $a, b \in R^{\dagger}$, then $R a=R b$ is equivalent to $a^{\dagger} a=b^{\dagger} b$;

(3) If $a, b \in R^{\oplus}$, then $a R=b R$ is equivalent to $a a^{\boxplus}=b b^{\oplus}$;

(4) If $a, b \in R_{\oplus}$, then $R a=R b$ is equivalent to $a_{\oplus} a=b_{\oplus} b$.

For an idempotent $p$ in a ring $R$, every $a \in R$ can be written as

$$
a=p a p+p a(1-p)+(1-p) a p+(1-p) a(1-p)
$$

or in matrix form

$$
\left[\begin{array}{cc}
\text { pap } & p a(1-p) \\
(1-p) a p & (1-p) a(1-p)
\end{array}\right]
$$

The decomposition (2.6) is known as the Pierce decomposition. Notice that if the idempotent $p$ is a projection, then the above matrix representation preserves the involution.

Lemma 2.4. Let $a, p, q \in R$. Then the following are equivalent:

(1) $a \in R^{\oplus}$;

(2) $\left[9\right.$, Theorem 3.5] there exists a projection $p$ such that $p a=0$ and $a+p \in R^{-1}$;

(3) $\left[14\right.$, Theorem 3.3] there exists a projection $q$ such that $q a=0$ and $a(1-q)+q \in R^{-1}$.

Under these equivalence, one has that these projections $p$ and $q$ are unique and $p=q=$ $1-a a^{\oplus}$.

We give a sketch of the proof of this lemma for the sake of completeness.

If $a \in R^{\oplus}$, define the projection $p=1-a a^{\oplus}$. From (2.5), it is evident that $p a=0$, and thus, the Pierce decomposition of $a$ with respect to $p$ is

$$
a=\left[\begin{array}{cc}
0 & 0 \\
a p & a(1-p)
\end{array}\right]
$$

hence

$$
a+p=\left[\begin{array}{cc}
p & 0 \\
a p & a(1-p)
\end{array}\right] \quad \text { and } \quad a(1-p)+p=\left[\begin{array}{cc}
p & 0 \\
0 & a(1-p)
\end{array}\right] .
$$

Observe that $a^{\boxplus} \in a a^{\boxplus} R a a^{\boxplus}=(1-p) R(1-p)$ and $a(1-p) a^{\boxplus}=1-p=a^{\boxplus} a(1-p)$. Hence $a^{\boxplus}$ is the inverse of $a(1-p)$ in the ring $(1-p) R(1-p)$, and thus, by $(2.8), a+p$ and $a(1-p)+p$ are invertible in $R$ because $p$ is a unit in the ring $p R p$.

If exists a projection $p$ such that $p a=0$, then the Pierce decomposition of $a, a+p$, and $a(1-p)+p$ with respect to $p$ are written in (2.7) and (2.8). Since $p$ is a unit in the ring $p R p$, the above decompositions prove that $a+p \in R^{-1} \Leftrightarrow a(1-p)+p \in R^{-1} \Leftrightarrow$ $a(1-p) \in[(1-p) R(1-p)]^{-1}$. Now, it is not difficult to prove that if $a+p \in R^{-1}$ or 
$a(1-p)+p \in R^{-1}$, then $a$ is core invertible and the inverse of $a(1-p)$ in $(1-p) R(1-p)$ is the core inverse of $a$.

Assume that $p_{1}$ and $p_{2}$ are two projections such that $p_{i} a=0$ and $a+p_{i} \in R^{-1}$ for $i=1,2$. By the proof of the previous paragraph, the inverse of $a\left(1-p_{i}\right)$ in $\left(1-p_{i}\right) R\left(1-p_{i}\right)$ is $a^{\boxplus}$ for $i=1,2$. Therefore, $\left(1-p_{i}\right) a^{\boxplus}=a^{\boxplus}$ and $a\left(1-p_{i}\right) a^{\boxplus}=1-p_{i}$ for $i=1,2$. It is deduced that $a a^{\oplus}=1-p_{i}$ for $i=1,2$.

From the sketch of the proof of Lemma 2.4, if $a \in R^{\oplus}$, then the matrix representations of $a, a+p$, and $a(1-p)+p$ with respect to $p=1-a a^{\boxplus}$ are written in (2.7) and (2.8).

Lemma 2.5. [6, Lemma 2] Let $R$ be any unitary ring and $e^{2}=e \in R$. Then exe $+1-e$ is invertible in $R$ if and only if exe is invertible in eRe with unit e, for all $x \in R$. If (exe) $)^{-e}$ denotes the inverse of exe in eRe, then we have

$$
(e x e)^{-e}=e(e x e+1-e)^{-1} e
$$

and

$$
(e x e+1-e)^{-1}=(e x e)^{-e}+1-e .
$$

Observe that if we represent the element $e x e+1-e$ of the above lemma respect the idempotent $e$, we have $e x e+1-e=\left[\begin{array}{cc}e x e & 0 \\ 0 & 1-e\end{array}\right]$, which makes this lemma clear. Recall that the unity of the corner ring $(1-e) R(1-e)$ is $1-e$.

The absorption law in a ring $R$ means that for two invertible elements $a, b \in R$, we have $a^{-1}(a+b) b^{-1}=a^{-1}+b^{-1}$. In [8], Jin and Benítez investigated the absorption law for the core inverse.

Lemma 2.6. [8, Theorem 3.5] Let $a, b \in R^{\boxplus}$. Then the following are equivalent:

(1) $a^{\boxplus}(a+b) b^{\oplus}=a^{\boxplus}+b^{\oplus} ;$

(2) $a R=b R$;

(3) ${ }^{\circ} a={ }^{\circ} b$.

In the following theorem, we will give more necessary and sufficient conditions such that the absorption law for the core inverse is valid. Let $a^{\pi}=1-a a^{\boxplus}$.

Theorem 2.7. Let $a, b \in R^{\oplus}$. Then the following are equivalent:

(1) $a a^{\oplus}=b b^{\oplus ;}$

(2) $a^{\pi}=b^{\pi}$;

(3) $a^{\boxplus}(a+b) b^{\oplus}=a^{\boxplus}+b^{\oplus} ;$

(4) $a^{\pi} b=0$ and $a^{\pi}+b \in R^{-1}$

(5) $a^{\pi} b=0$ and $a^{\pi}+b\left(1-a^{\pi}\right) \in R^{-1}$

(6) $a^{\pi} b=0$ and $b\left(1-a^{\pi}\right)$ is invertible in $\left(1-a^{\pi}\right) R\left(1-a^{\pi}\right)$. 
In this case, the expression of the subset of elements $b$ such that $a^{\pi}=b^{\pi}$ is

$$
\left\{b \in R: a^{\pi}=b^{\pi}\right\}=\left\{z+t: z \in\left(1-a^{\pi}\right) R a^{\pi}, t \in\left[\left(1-a^{\pi}\right) R\left(1-a^{\pi}\right)\right]^{-1}\right\} .
$$

Moreover, the relationship of $a^{\pi}+b$ and $a^{\pi}+b\left(1-a^{\pi}\right)$ is

$$
\left(a^{\pi}+b\right)^{-1}=\left(b^{2}+a^{\pi}\right)^{-1}\left[a^{\pi}+b\left(1-a^{\pi}\right)\right] .
$$

Proof. (1) $\Leftrightarrow(2)$ is trivial.

$(1) \Leftrightarrow(3)$ follows from Corollary 2.3 and Lemma 2.6.

$(1) \Rightarrow(4)$. Suppose $a a^{\boxplus}=b b^{\oplus}$, or equivalently $a^{\pi}=b^{\pi}$ since (1) and (2) are equivalent. Taking into account the equality given in $(2)$, we have $a^{\pi} b=\left(1-a a^{\boxplus}\right) b=\left(1-b b^{\boxplus}\right) b=0$. Since

$\left(a^{\pi}+b\right)\left(b^{\oplus}+1-b^{\oplus} b\right)=\left(1-a a^{\boxplus}+b\right)\left(b^{\oplus}+1-b^{\oplus} b\right)=\left(1-b b^{\oplus}+b\right)\left(b^{\oplus}+1-b^{\oplus} b\right)=1$

and

$$
\left(b^{\oplus}+1-b^{\oplus} b\right)\left(a^{\pi}+b\right)=\left(b^{\oplus}+1-b^{\oplus} b\right)\left(1-b b^{\oplus}+b\right)=1,
$$

we have that $a^{\pi}+b$ is invertible.

$(4) \Rightarrow(2)$ (or $(6) \Rightarrow(2))$. It is easy to check that $a^{\pi}$ is a projection. By Lemma 2.4, we have $a^{\pi}=1-b b^{\oplus}=b^{\pi}$.

$(1) \Rightarrow(5)$. Suppose $a a^{\boxplus}=b b^{\oplus}$. Then

$$
\begin{aligned}
a^{\pi} b & =\left(1-a a^{\oplus}\right) b=\left(1-b b^{\oplus}\right) b=0 ; \\
{\left[a^{\pi}+b\left(1-a^{\pi}\right)\right]\left(b^{\oplus}+1-b b^{\oplus}\right) } & =\left(1-b b^{\oplus}+b^{2} b^{\oplus}\right)\left(b^{\oplus}+1-b b^{\oplus}\right)=1 ; \\
\left(b^{\oplus}+1-b b^{\oplus}\right)\left[a^{\pi}+b\left(1-a^{\pi}\right)\right] & =\left(b^{\oplus}+1-b b^{\oplus}\right)\left(1-b b^{\oplus}+b^{2} b^{\oplus}\right)=1 .
\end{aligned}
$$

Thus $a^{\pi}+b\left(1-a^{\pi}\right)$ is invertible.

$(5) \Leftrightarrow(6)$ By $a^{\pi} b=0$, we have $a^{\pi}+b\left(1-a^{\pi}\right)=a^{\pi}+\left(1-a^{\pi}\right) b\left(1-a^{\pi}\right)$. Thus by Lemma 2.5, we get the equivalence between (5) and (6).

Now, we will find the general expression of the elements $b \in R^{\boxplus}$ such that $a a^{\boxplus}=b b^{\oplus}$. We use the matrix representations of $a^{\pi}$ and $b$ with respect to the projection $a^{\pi}$. Then $a^{\pi}=\left[\begin{array}{cc}a^{\pi} & 0 \\ 0 & 0\end{array}\right]$. Let

$$
b=\left[\begin{array}{ll}
x & y \\
z & t
\end{array}\right]
$$

satisfy $a a^{\boxplus}=b b^{\boxplus}$ (and therefore, also (2), (3) and (4) hold). Then $a^{\pi} b=0$ gives

$$
0=a^{\pi} b=\left[\begin{array}{cc}
a^{\pi} & 0 \\
0 & 0
\end{array}\right]\left[\begin{array}{ll}
x & y \\
z & t
\end{array}\right] \Rightarrow x=y=0
$$

Thus

$$
a^{\pi}+b=\left[\begin{array}{ll}
a^{\pi} & 0 \\
z & t
\end{array}\right] \Rightarrow t \in\left[\left(1-a^{\pi}\right) R\left(1-a^{\pi}\right)\right]^{-1},
$$

because $a^{\pi}$, the $(1,1)$ entry of the last representation, is a unit in the corner $\operatorname{ring} a^{\pi} R a^{\pi}$. Also, it is simple to see that

$$
c=\left[\begin{array}{ll}
0 & 0 \\
z & t
\end{array}\right], t \in\left[\left(1-a^{\pi}\right) R\left(1-a^{\pi}\right)\right]^{-1} \quad \Rightarrow \quad a^{\pi} c=0 \text { and } a^{\pi}+c \in R^{-1} .
$$


Hence we have proved the characterisation of the elements $b$ such that $a^{\pi}=b^{\pi}$ stated in the theorem.

Last, we give the relationship of $a^{\pi}+b$ and $a^{\pi}+b\left(1-a^{\pi}\right)$. Since $a^{\pi}=b^{\pi}$, we have $a^{\pi}+b=b^{\pi}+b$ and $a^{\pi}+b\left(1-a^{\pi}\right)=b^{\pi}+b\left(1-b^{\pi}\right)$. Thus

$$
\left[a^{\pi}+b\left(1-a^{\pi}\right)\right]\left(a^{\pi}+b\right)=\left[b^{\pi}+b\left(1-b^{\pi}\right)\right]\left(b^{\pi}+b\right)=b^{2}+b^{\pi}=b^{2}+a^{\pi},
$$

which is invertible because $a^{\pi}+b$ and $a^{\pi}+b\left(1-a^{\pi}\right)$ are both invertible. Thus

$$
\left(a^{\pi}+b\right)^{-1}=\left(b^{2}+a^{\pi}\right)^{-1}\left[a^{\pi}+b\left(1-a^{\pi}\right)\right] .
$$

Observe that the condition (1) of Theorem 2.7 is symmetric in $a$ and in $b$. Hence we can get duplicate results.

Theorem 2.8. Let $a, b \in R^{\oplus}$. Then the following are equivalent:

(1) $a a^{\oplus}=b b^{\oplus} ;$

(2) $b^{\pi}=a^{\pi} b^{\pi}$ and $b^{\pi}+1-a^{\pi}$ is left invertible;

(3) $1-a^{\pi}=\left(1-a^{\pi}\right)\left(1-b^{\pi}\right)$ and $b^{\pi}+1-a^{\pi}$ is left invertible;

(4) $\left[a^{\pi}, b^{\pi}\right]=0, b^{\pi}+1-a^{\pi}$ is right invertible and $a^{\pi}+1-b^{\pi}$ is left invertible;

(5) $a^{\pi} b^{\pi}$ is Hermitian, $b^{\pi}+1-a^{\pi}$ is right invertible and $a^{\pi}+1-b^{\pi}$ is left invertible.

Proof. The implications $(1) \Rightarrow(2)$ and $(1) \Rightarrow(4)$ are trivial.

$(2) \Rightarrow(1)$. Suppose that $b^{\pi}=a^{\pi} b^{\pi}$ and $u=b^{\pi}+1-a^{\pi}$ is left invertible. Then we have

$$
r u=1 \quad \text { for some } r \in R \text {. }
$$

Since $a^{\pi}$ and $b^{\pi}$ are Hermitian, then $a^{\pi} b^{\pi}=b^{\pi}$ implies $b^{\pi} a^{\pi}=b^{\pi}$. Thus

$$
\begin{gathered}
u\left(1-a^{\pi}\right)=\left(b^{\pi}+1-a^{\pi}\right)\left(1-a^{\pi}\right)=b^{\pi}\left(1-a^{\pi}\right)+\left(1-a^{\pi}\right)^{2}=1-a^{\pi} \\
u\left(1-b^{\pi}\right)=\left(b^{\pi}+1-a^{\pi}\right)\left(1-b^{\pi}\right)=b^{\pi}\left(1-b^{\pi}\right)+\left(1-a^{\pi}\right)\left(1-b^{\pi}\right)=1-a^{\pi} .
\end{gathered}
$$

By equations (2.9), (2.10) and (2.11), we have

$$
1-a^{\pi}=r u\left(1-a^{\pi}\right)=r\left(1-a^{\pi}\right)=r u\left(1-b^{\pi}\right)=1-b^{\pi} .
$$

That is $a a^{\oplus}=b b^{\oplus}$.

$(2) \Leftrightarrow(3)$. Since $\left(1-a^{\pi}\right)\left(1-b^{\pi}\right)=1-a^{\pi}-b^{\pi}+a^{\pi} b^{\pi}$, then it is obvious that $a^{\pi} b^{\pi}=b^{\pi}$ if and only if $\left(1-a^{\pi}\right)\left(1-b^{\pi}\right)=1-a^{\pi}$.

(4) $\Leftrightarrow(5)$. If $\left[a^{\pi}, b^{\pi}\right]=0$, then $\left(a^{\pi} b^{\pi}\right)^{*}=\left(b^{\pi} a^{\pi}\right)^{*}=a^{\pi} b^{\pi}$ and reciprocally.

(4) $\Rightarrow(1)$. Assume $\left[a^{\pi}, b^{\pi}\right]=0$ and $u=b^{\pi}+1-a^{\pi}$ is right invertible and $v=a^{\pi}+1-b^{\pi}$ is left invertible. We have

$$
u s=1 \text { and } t v=1 \quad \text { for some } s, t \in R .
$$


From $a^{\pi} b^{\pi}=b^{\pi} a^{\pi}$, we get

$$
\left(1-b^{\pi}\right) u=\left(1-b^{\pi}\right)\left(b^{\pi}+1-a^{\pi}\right)=\left(1-b^{\pi}\right) b^{\pi}+\left(1-b^{\pi}\right)\left(1-a^{\pi}\right)=\left(1-b^{\pi}\right)\left(1-a^{\pi}\right) .
$$

As $a^{\pi}$ and $b^{\pi}$ commute, so does $1-a^{\pi}$ and $1-b^{\pi}$. Thus from (2.13) we get

$$
\left(1-b^{\pi}\right)\left(1-a^{\pi}\right) u=\left(1-b^{\pi}\right)\left(1-a^{\pi}\right) .
$$

From equations (2.12), (2.13) and (2.14), we have

$$
1-b^{\pi}=\left(1-b^{\pi}\right) u s=\left(1-b^{\pi}\right)\left(1-a^{\pi}\right) s=\left(1-b^{\pi}\right)\left(1-a^{\pi}\right) u s=\left(1-b^{\pi}\right)\left(1-a^{\pi}\right) .
$$

Similarly,

$$
v\left(1-a^{\pi}\right)=\left(a^{\pi}+1-b^{\pi}\right)\left(1-a^{\pi}\right)=a^{\pi}\left(1-a^{\pi}\right)+\left(1-b^{\pi}\right)\left(1-a^{\pi}\right)=\left(1-b^{\pi}\right)\left(1-a^{\pi}\right),
$$

and

$$
v\left(1-b^{\pi}\right)\left(1-a^{\pi}\right)=\left(1-b^{\pi}\right)\left(1-a^{\pi}\right) .
$$

From equations (2.12), (2.16) and (2.17), we have

$$
1-a^{\pi}=t\left(1-b^{\pi}\right)\left(1-a^{\pi}\right)=\left(1-b^{\pi}\right)\left(1-a^{\pi}\right) .
$$

By $(2.15)$ and (2.18), we have $a a^{\boxplus}=b b^{\oplus}$.

Let $a \in R^{\boxplus}$. If $a a^{*}+1-a a^{\boxplus} \in R^{-1}$, then $a=\left(a a^{*}+1-a a^{\boxplus}\right)^{-1} a a^{*} a \in R a a^{*} a$, which implies that $a$ is Moore-Penrose invertible in view of [16, Theorem 2.16]. Therefore, if $a \notin R^{\dagger}$, then $a a^{*}+1-a a^{\oplus}$ is not invertible.

Lemma 2.9. Let $a \in R^{\boxplus}$. If $a a^{\pi} a^{*}=0$, then $a a^{*}+a^{\pi} \in R^{-1}$.

Proof. We use the matrix representation of $a$ with respect to the projection $a^{\pi}$.

$$
a=\left[\begin{array}{cc}
0 & 0 \\
a a^{\pi} & a\left(1-a^{\pi}\right)
\end{array}\right], a^{\pi}=\left[\begin{array}{cc}
a^{\pi} & 0 \\
0 & 0
\end{array}\right], a^{*}=\left[\begin{array}{cc}
0 & a^{\pi} a^{*} \\
0 & \left(1-a^{\pi}\right) a^{*}
\end{array}\right], a a^{*}=\left[\begin{array}{cc}
0 & 0 \\
0 & a a^{*}
\end{array}\right] .
$$

It is easy to check that $a\left(1-a^{\pi}\right)$ is invertible in $\left(1-a^{\pi}\right) R\left(1-a^{\pi}\right)$ with the inverse $a^{\boxplus}$ and $a a^{\pi} a^{*}=0 \Leftrightarrow a\left(1-a^{\pi}\right) a^{*}=a a^{*} \Leftrightarrow a\left(1-a^{\pi}\right)\left(1-a^{\pi}\right) a^{*}=a a^{*} \Leftrightarrow a\left(1-a^{\pi}\right)\left(a\left(1-a^{\pi}\right)\right)^{*}=a a^{*}$.

Thus $a a^{*}$ is invertible in $\left(1-a^{\pi}\right) R\left(1-a^{\pi}\right)$ because $a a^{*}$ is the product of two invertible elements in $\left(1-a^{\pi}\right) R\left(1-a^{\pi}\right)$. Since $a a^{*}$ is invertible in $\left(1-a^{\pi}\right) R\left(1-a^{\pi}\right)$ if and only if $a a^{*}+a^{\pi} \in R^{-1}$, we finish the proof.

Let $a \in R^{\oplus}$. If $a a^{\pi}=0$, then $a=a^{2} a^{\boxplus}$, and therefore, $a^{\boxplus} a=a^{\boxplus}\left(a^{2} a^{\boxplus}\right)=\left(a^{\boxplus} a^{2}\right) a^{\boxplus}=$ $a a^{\oplus}$, thus $a$ is Moore-Penrose invertible, $a^{\dagger}=a^{\oplus}$, and $a$ is EP by [12, Theorem 3.1]. Reciprocally, it is evident that if $a$ is EP, then $a^{\boxplus}=a^{\dagger}$ and $a a^{\boxplus}=a^{\oplus} a$, which implies $a a^{\pi}=0$. Notice that the condition $a a^{\pi} a^{*}=0$ is weaker than $a a^{\pi}=0$. But, it is strictly weaker, as the following example shows. 
Example 2.10. Let $R$ be the ring of $2 \times 2$ matrices whose entries are in $\mathbb{Z}_{4}$. In $R$ we take the matrix transposition as the involution. Consider $a=\left[\begin{array}{ll}1 & 2 \\ 0 & 0\end{array}\right]$. By checking the (1.1) and (1.2), it is simple to prove

$$
a^{\dagger}=\left[\begin{array}{ll}
1 & 0 \\
2 & 0
\end{array}\right], \quad a^{\oplus}=\left[\begin{array}{ll}
1 & 0 \\
0 & 0
\end{array}\right] .
$$

Now, it is simple to compute

$$
a a^{\dagger}=\left[\begin{array}{ll}
1 & 0 \\
0 & 0
\end{array}\right], \quad a^{\dagger} a=\left[\begin{array}{ll}
1 & 2 \\
2 & 0
\end{array}\right],
$$

which, by the definition of EP element, shows that $a$ is not EP. In addition,

$$
a^{\pi}=1-a a^{\oplus}=\left[\begin{array}{ll}
0 & 0 \\
0 & 1
\end{array}\right], \quad a a^{\pi} a^{*}=\left[\begin{array}{ll}
1 & 2 \\
0 & 0
\end{array}\right]\left[\begin{array}{ll}
0 & 0 \\
0 & 1
\end{array}\right]\left[\begin{array}{ll}
1 & 0 \\
2 & 0
\end{array}\right]=0 .
$$

But if the ring $R$ is $*$-reducing, then $a a^{\pi} a^{*}=0$ implies that $a a^{\pi}=0$ (recall that a ring $R$ is $*$-reducing if $x^{*} x=0$ implies $x=0$ for any $\left.x \in R\right)$. In fact: $0=a a^{\pi} a^{*}=a a^{\pi}\left(a a^{\pi}\right)^{*}$ implies $a a^{\pi}=0$. Of course, the ring considered in the above example is not reducing: it is enough to take $x=\left[\begin{array}{ll}2 & 2 \\ 2 & 2\end{array}\right]$ to see that $x x^{*}=0$ and $x \neq 0$.

Theorem 2.11. Let $a, b \in R^{\oplus}$ with $a a^{\pi} a^{*}=0$. Then $a a^{\boxplus}=b b^{\oplus}$ if and only if $b^{\pi}=a^{\pi} b^{\pi}$ and $u=a a^{*}+b^{\pi}$ is invertible.

Proof. We will prove the necessity and the sufficiency simultaneously. Since $a a^{\pi} a^{*}=0$, by Lemma 2.9 , we have that $a a^{*}+a^{\pi}$ is invertible. Notice that $a a^{\boxplus}=b b^{\oplus}$, i.e., $a^{\pi}=b^{\pi}$, implies $a^{\pi} b^{\pi}=b^{\pi}$. Furthermore, we can use $a^{\pi} b^{\pi}=b^{\pi}$ to prove both the necessity and the sufficiency. Taking involution in $a^{\pi} b^{\pi}=b^{\pi}$ we get $b^{\pi} a^{\pi}=b^{\pi}$. Observe that

$$
\begin{aligned}
\left(b^{\pi}+1-a^{\pi}\right)\left(a a^{*}+a^{\pi}\right) & =b^{\pi} a a^{*}+b^{\pi} a^{\pi}+\left(1-a^{\pi}\right) a a^{*}+\left(1-a^{\pi}\right) a^{\pi} \\
& =b^{\pi} a a^{\boxplus} a a^{*}+b^{\pi}+a a^{*} \\
& =b^{\pi}\left(1-a^{\pi}\right) a a^{*}+b^{\pi}+a a^{*} \\
& =b^{\pi}+a a^{*} .
\end{aligned}
$$

And, therefore, $b^{\pi}+1-a^{\pi}=\left(b^{\pi}+a a^{*}\right)\left(a a^{*}+a^{\pi}\right)^{-1}$. Now, by Theorem 2.8 and using that a Hermitian element is left invertible if and only if such element is invertible, we have:

$$
a a^{\boxplus}=b b^{\boxplus} \Leftrightarrow b^{\pi}=a^{\pi} b^{\pi} \text { and } b^{\pi}+1-a^{\pi} \in R^{-1} \Leftrightarrow b^{\pi}=a^{\pi} b^{\pi} \text { and } b^{\pi}+a a^{*} \in R^{-1} .
$$

The proof is finished.

\section{Projections $a^{(1,2,4)} a$ and $b b^{(1,2,3)}$}

In this section, we will investigate projections $a^{(1,2,4)} a$ and $b b^{(1,2,3)}$. If we replace $a^{(1,2,4)}$ and $b^{(1,2,3)}$ by $a^{\dagger}$ and $b^{\dagger}$, respectively, then we can get some special corollaries, which will be useful when we discuss the projection equation $a^{\dagger} a=b b^{\dagger}$. 
Lemma 3.1. Let $a \in R$. Then we have the following results:

(1) If $a \in R^{\{1,2,3\}}$ and $a^{(1,2,3)} \in a\{1,2,3\}$, then $a^{(1,2,3)} b=a^{(1,2,3)} c$ if and only if $a^{*} b=a^{*} c$ for all $b, c \in R$;

(2) If $a \in R^{\{1,2,4\}}$ and $a^{(1,2,4)} \in a\{1,2,4\}$, then $b a^{(1,2,4)}=c a^{(1,2,4)}$ if and only if $b a^{*}=c a^{*}$ for all $b, c \in R$.

Proof. Since the proof of (2) is similar to the proof of (1), we only prove (1). Since $a \in R^{\{1,2,3\}}$, then

$$
\begin{aligned}
a^{(1,2,3)} b & =a^{(1,2,3)} a a^{(1,2,3)} b=a^{(1,2,3)}\left(a^{(1,2,3)}\right)^{*} a^{*} b=a^{(1,2,3)}\left(a^{(1,2,3)}\right)^{*} a^{*} c=a^{(1,2,3)} c ; \\
a^{*} b & =\left(a a^{(1,2,3)} a\right)^{*} b=a^{*} a a^{(1,2,3)} b=a^{*} a a^{(1,2,3)} c=a^{*} c .
\end{aligned}
$$

Proposition 3.2. Let $a \in R^{\{1,2,4\}}, b \in R^{\{1,2,3\}}$ and $a^{(1,2,4)} \in a\{1,2,4\}, b^{(1,2,3)} \in b\{1,2,3\}$. Then $a b b^{(1,2,3)} a^{(1,2,4)} a b=a b$ if and only if $b^{(1,2,3)} a^{(1,2,4)} a b b^{(1,2,3)} a^{(1,2,4)}=b^{(1,2,3)} a^{(1,2,4)}$.

Proof. By Lemma 3.1, we have

$$
\begin{aligned}
a b b^{(1,2,3)} a^{(1,2,4)} a b=a b & \Leftrightarrow b^{*}\left(a^{(1,2,4)} a\right)^{*}\left(b b^{(1,2,3)}\right)^{*} a^{*}=b^{*} a^{*} \\
& \Leftrightarrow b^{*} a^{(1,2,4)} a b b^{(1,2,3)} a^{*}=b^{*} a^{*} \\
& \Leftrightarrow b^{(1,2,3)} a^{(1,2,4)} a b b^{(1,2,3)} a^{(1,2,4)}=b^{(1,2,3)} a^{(1,2,4)} .
\end{aligned}
$$

Corollary 3.3. Let $a, b \in R^{\dagger}$. Then $a b b^{\dagger} a^{\dagger} a b=a b$ if and only if $b^{\dagger} a^{\dagger} a b b^{\dagger} a^{\dagger}=b^{\dagger} a^{\dagger}$.

Lemma 3.4. Let $R$ be $a$ *-reducing ring with $p^{*}=p=p^{2} \in R$ and $q^{*}=q=q^{2} \in R$. Then the following are equivalent:

(1) $(p q)^{2}=p q$;

(2) $(q p)^{2}=q p$;

(3) $p q=q p$

Proof. (1) $\Rightarrow(3)$ We use the matrix representations of $p$ and $q$ with respect to the projection $p$. Then $p=\left[\begin{array}{ll}p & 0 \\ 0 & 0\end{array}\right]$ and $q=\left[\begin{array}{ll}q_{1} & q_{2} \\ q_{2}^{*} & q_{3}\end{array}\right]$ by $q^{*}=q$. Then $p q=\left[\begin{array}{cc}q_{1} & q_{2} \\ 0 & 0\end{array}\right]$ and $q p=\left[\begin{array}{ll}q_{1} & 0 \\ q_{2}^{*} & 0\end{array}\right]$. Now $(p q)^{2}=(p q)$ and $q^{2}=q$ implies, respectively, $q_{1}^{2}=q_{1}$ and $q_{1}^{2}+q_{2} q_{2}^{*}=q_{1}$. Hence, we have $q_{2} q_{2}^{*}=0$, which implies $q_{2}=0$ because $R$ is a $*$-reducing ring. Thus $p q=\left[\begin{array}{cc}q_{1} & 0 \\ 0 & 0\end{array}\right]$ and $q p=\left[\begin{array}{cc}q_{1} & 0 \\ 0 & 0\end{array}\right]$. That is $p q=q p$.

$(3) \Rightarrow(1)$ and $(3) \Rightarrow(2)$ are trivial. By the symmetry of $p$ and $q$, we have $(2) \Rightarrow(3)$ with the help of $(1) \Rightarrow(3)$. 
Example 3.5. In a general ring $R$, the implication of $(p q)^{2}=p q \Rightarrow p q=q p$ may not hold, where $p^{*}=p=p^{2} \in R$ and $q^{*}=q=q^{2} \in R$. Let us consider the following counterexample. Let $R$ be the ring of $2 \times 2$ matrices over $\mathbb{Z}_{4}$ with the conjugate transposition as involution. Considering the matrices $p=\left[\begin{array}{ll}1 & 0 \\ 0 & 0\end{array}\right]$ and $q=\left[\begin{array}{ll}1 & 2 \\ 2 & 0\end{array}\right]$, it is easy to check that $p^{*}=p=p^{2}$ and $q^{*}=q=q^{2}$ and $(p q)^{2}=p q$, and yet $p q=\left[\begin{array}{ll}1 & 2 \\ 0 & 0\end{array}\right]$ and $q p=\left[\begin{array}{ll}1 & 0 \\ 2 & 0\end{array}\right]$.

Theorem 3.6. Let $R$ be $a *$-reducing ring. If $a \in R^{\{1,2,4\}}, b \in R^{\{1,2,3\}}$ and $a^{(1,2,4)} \in$ $a\{1,2,4\}, b^{(1,2,3)} \in b\{1,2,3\}$. Then the following are equivalent:

(1) $a b b^{(1,2,3)} a^{(1,2,4)} a b=a b$;

(2) $\left[a^{(1,2,4)} a, b b^{(1,2,3)}\right]=0$;

(3) $b^{(1,2,3)} a^{(1,2,4)} a b b^{(1,2,3)} a^{(1,2,4)}=b^{(1,2,3)} a^{(1,2,4)}$.

Proof. (3) $\Rightarrow(2)$. By Proposition 3.2, we have $a b b^{(1,2,3)} a^{(1,2,4)} a b=a b$ if and only if $b^{(1,2,3)} a^{(1,2,4)} a b b^{(1,2,3)} a^{(1,2,4)}=b^{(1,2,3)} a^{(1,2,4)}$. Define the projections $e=a^{(1,2,4)} a$ and $f=$ $b b^{(1,2,3)}$. Multiplying by $a^{(1,2,4)}$ on the left side of $a b b^{(1,2,3)} a^{(1,2,4)} a b=a b$ and multiplying by $b^{(1,2,3)}$ on the right side of $a b b^{(1,2,3)} a^{(1,2,4)} a b=a b$, then we have $(e f)^{2}=e f$, thus $\left[a^{(1,2,4)} a, b b^{(1,2,3)}\right]=0$ by Lemma 3.4 .

$(2) \Rightarrow(1)$ is trivial.

$(1) \Rightarrow(3)$. It is obvious by Proposition 3.2.

Corollary 3.7. [10, Theorem 2.1] Let $R$ be a -reducing ring with $a, b \in R^{\dagger}$. Then the following are equivalent:

(1) $a b b^{\dagger} a^{\dagger} a b=a b$;

(2) $\left[a^{\dagger} a, b b^{\dagger}\right]=0$;

(3) $b^{\dagger} a^{\dagger} a b b^{\dagger} a^{\dagger}=b^{\dagger} a^{\dagger}$.

Lemma 3.8. [13, Lemma 8] Let $a, b \in R$. Then:

(1) $a R \subseteq b R$ implies ${ }^{\circ} b \subseteq{ }^{\circ} a$ and the converse is valid whenever $b$ is regular;

(2) $R a \subseteq R b$ implies $b^{\circ} \subseteq a^{\circ}$ and the converse is valid whenever $b$ is regular.

Theorem 3.9. Let $R$ be a $*$-reducing ring with $a, b \in R^{\dagger}$. Then the following are equivalent:

(1) $a^{\dagger} a=b b^{\dagger}$;

(2) $\left[a^{\dagger} a, b b^{\dagger}\right]=0, a R \subseteq a b R$ and $R b \subseteq R a b$;

(3) $(a b)^{\dagger}=b^{\dagger} a^{\dagger}, a R \subseteq a b R$ and $R b \subseteq R a b ;$

(4) $a b b^{\dagger} a^{\dagger} a b=a b, a R \subseteq a b R$ and $R b \subseteq R a b$;

(5) $b^{\dagger} a^{\dagger} a b b^{\dagger} a^{\dagger}=b^{\dagger} a^{\dagger}, a R \subseteq a b R$ and $R b \subseteq R a b$; 
(6) $\left[a^{\dagger} a, b b^{\dagger}\right]=0,{ }^{\circ}(a b) \subseteq{ }^{\circ} a$ and $(a b)^{\circ} \subseteq b^{\circ}$;

(7) $(a b)^{\dagger}=b^{\dagger} a^{\dagger},{ }^{\circ}(a b) \subseteq{ }^{\circ} a$ and $(a b)^{\circ} \subseteq b^{\circ}$;

(8) $a b b^{\dagger} a^{\dagger} a b=a b,{ }^{\circ}(a b) \subseteq{ }^{\circ} a$ and $(a b)^{\circ} \subseteq b^{\circ}$;

(9) $b^{\dagger} a^{\dagger} a b b^{\dagger} a^{\dagger}=b^{\dagger} a^{\dagger},{ }^{\circ}(a b) \subseteq{ }^{\circ} a$ and $(a b)^{\circ} \subseteq b^{\circ}$.

Proof. The equivalence between (2), (4) and (5) can be obtained by Corollary 3.3 and Corollary 3.7.

(1) $\Rightarrow(2)$. Suppose $a^{\dagger} a=b b^{\dagger}$. Then

$$
\begin{aligned}
{\left[a^{\dagger} a, b b^{\dagger}\right] } & =\left[a^{\dagger} a, a^{\dagger} a\right]=0 \\
a & =a a^{\dagger} a=a b b^{\dagger} \\
b & =b b^{\dagger} b=a^{\dagger} a b
\end{aligned}
$$

that is $\left[a^{\dagger} a, b b^{\dagger}\right]=0, a R \subseteq a b R$ and $R b \subseteq R a b$.

$(2) \Rightarrow(3)$. By Corollary 3.7, we have $\left[a^{\dagger} a, b b^{\dagger}\right]=0 \Leftrightarrow a b b^{\dagger} a^{\dagger} a b=a b$ and $b^{\dagger} a^{\dagger} a b b^{\dagger} a^{\dagger}=$ $b^{\dagger} a^{\dagger}$. Since $a R \subseteq a b R$ and $R b \subseteq R a b$, then

$$
\begin{aligned}
& a=a b x \text { for some } x \in R \\
& b=y a b \text { for some } y \in R .
\end{aligned}
$$

Thus by equations (3.1) and (3.2), we have

$$
\begin{aligned}
& a=a b b^{\dagger} a^{\dagger} a b x=a b b^{\dagger} a^{\dagger} a=a a^{\dagger} a b b^{\dagger}=a b b^{\dagger} ; \\
& b=y a b b^{\dagger} a^{\dagger} a b=b b^{\dagger} a^{\dagger} a b=a^{\dagger} a b .
\end{aligned}
$$

The equations (3.3) and (3.4) give that

$$
\begin{aligned}
& a b b^{\dagger} a^{\dagger}=a a^{\dagger} \text { is Hermitian; } \\
& b^{\dagger} a^{\dagger} a b=b^{\dagger} b \text { is Hermitian. }
\end{aligned}
$$

$(3) \Rightarrow(1)$. Suppose $(a b)^{\dagger}=b^{\dagger} a^{\dagger}, a R \subseteq a b R$, and $R b \subset R a b$. There exist $x, y \in R$ such that $a=a b x$ and $b=y a b$. Now, $a b b^{\dagger} a^{\dagger} a=a b b^{\dagger} a^{\dagger} a b x=a b(a b)^{\dagger} a b x=a b x=a$, and similarly, $b b^{\dagger} a^{\dagger} a b=y a b(a b)^{\dagger} a b=y a b=b$. Thus

$$
b b^{\dagger} R=b b^{\dagger} a^{\dagger} a R \text { and } a^{\dagger} a R=a^{\dagger} a b b^{\dagger} R .
$$

The condition $(a b)^{\dagger}=b^{\dagger} a^{\dagger}$ gives $\left(a^{\dagger} a b b^{\dagger}\right)^{2}=a^{\dagger} a b b^{\dagger}$. By Lemma 3.4 we get $a^{\dagger} a b b^{\dagger}=b b^{\dagger} a^{\dagger} a$. Therefore we have $a^{\dagger} a=b b^{\dagger}$ by equation (3.5) and Corollary 2.2.

$(2) \Leftrightarrow(6)$. It is easy to see that by Lemma 3.8. The proofs of $(3) \Leftrightarrow(7),(4) \Leftrightarrow(8)$ and $(5) \Leftrightarrow(9)$ are similar to the proof of $(2) \Leftrightarrow(6)$.

The element $a \in R^{\dagger}$ is said to be $b i$-EP when $\left[a^{\dagger} a, a a^{\dagger}\right]=0$. Let $a$ be an element of an associative ring $R$ with 1 . In [4, Proposition 8.22], we have $a$ has a group inverse if and only if $a^{2} x=a$ and $y a^{2}=a$ both have solutions. Thus, taking $a=b$ in Theorem 3.9, we get the following corollary. 
Corollary 3.10. Let $R$ be $a *$-reducing ring with $a \in R^{\dagger}$. Then the following are equivalent:

(1) $a \in R^{\mathrm{EP}}$;

(2) $a \in R^{\#}$ and $a$ is bi-EP;

(3) $a \in R^{\#}$ and $\left(a^{\dagger}\right)^{2}=\left(a^{2}\right)^{\dagger}$;

(4) $a \in R^{\#}$ and $\left(a^{\dagger}\right)^{2} \in a^{2}\{1\}$;

(5) $a \in R^{\#}$ and $a^{2} \in\left(a^{\dagger}\right)^{2}\{1\}$.

\section{ACKNOWLEDGMENTS}

This research is supported by the National Natural Science Foundation of China (No. 11371089). The first author is grateful to China Scholarship Council for giving him a scholarship for his further study in Universitat Politècnica de València, Spain.

We are very grateful to the referees for their carefully reading of the preliminary version of the manuscript.

\section{References}

[1] Baksalary OM, Trenkler G. Core inverse of matrices. Linear Multilinear Algebra. 2010; 58(6):681-697.

[2] Benítez J. Moore-Penrose inverses and commuting elements of $C^{*}$-algebras. J. Math. Anal. Appl. 2008;345(2):766-770.

[3] Berberian SK. Baer *-rings. Springer-Verlag; 1972.

[4] Bhaskara Rao KPS. The Theory of Generalized Inverses Over Commutative Rings. Taylor and Francis; 2002.

[5] Hartwig RE. Block generalized inverses. Arch. Retion. Mech. Anal. 1976;61(3):197251.

[6] Huylebrouck D, Puystjens R, Van Geel J. The Moore-Penrose inverse of a matrix over a semisimple Artinian ring. Linear and Multilinear Algebra. 1984;16(1-4):239-246.

[7] Hartwig RE, Spindelböck K. Matrices for which $A^{*}$ and $A^{\dagger}$ commute. Linear Multilinear Algebra. 1983;14(3):241-256.

[8] Jin HW, Benítez J. The absorption laws for the generalized inverses in rings. Electron. J. Linear Algebra. 2015;30:827-842.

[9] Li TT, Chen JL. Characterizations of core and dual core inverse in rings with involution. Linear Multilinear Algebra. doi.org/10.1080/03081087.2017.1320963.

[10] Mosić D, Djordjević DS. Reverse order law for the Moore-Penrose inverse in $C^{*}$ algebras. Electron. J. Linear Algebra. 2011;22:92-111. 
[11] Patrício P, Mendes Araújo C. Moore-Penrose invertibility in involutory rings: the case $a a^{\dagger}=b b^{\dagger}$. Linear Multilinear Algebra. 2010;58(4):445-452.

[12] Rakić DS, Dinčić N, Djordjević DS. Group, Moore-Penrose, core and dual core inverse in rings with involution. Linear Algebra Appl. 2014;463:115-133.

[13] von Neumann J. On regular rings. Proc. Natl. Acad. Sci. U.S.A. 1936;22(12):707-713.

[14] Xu SZ, Chen JL, Benítez J. EP elements in rings with involution. arXiv:1602. 08184[math.RA].

[15] Xu SZ, Chen JL, Zhang XX, New characterizations for core and dual core inverses in rings with involution. Front. Math. China. 2017;12(1):231-246.

[16] Zhu HH, Chen JL, Patrício P. Further results on the inverse along an element in semigroups and rings. Linear Multilinear Algebra. 2016; 64 (3) 393-403. 